\title{
Radiographic patterns of osteoarthritis of the knee joint in the community: the importance of the patellofemoral joint
}

\author{
T E McAlindon, S Snow, C Cooper, P A Dieppe
}

\begin{abstract}
The intimate relation which the patella has with the knee joint and quadriceps muscle suggests that patellofemoral joint osteoarthritis is likely to be an important cause of knee pain and disability. Two hundred and seventy three subjects who reported knee pain in a postal questionnaire survey and 240 control subjects consented to have anteroposterior weightbearing and lateral knee radiographs. Each subject completed a Stanford Health Assessment Questionnaire (HAQ).

Radiographic knee osteoarthritis was found in $53 \%$ of symptomatic and $17 \%$ of asymptomatic subjects. Three patterns predominated patellofemoral, medial, and medial/patellofemoral joint disease in 11,21 , and $7 \%$ of the men and in $24,12,6 \%$ of the women respectively. The occurrence of isolated symptomatic patellofemoral joint osteoarthritis in this sample aged more than $\mathbf{5 5}$ years was estimated as $8 \%$ in women and $2 \%$ in men. All patterns of symptomatic knee joint osteoarthritis increased with age in women but peaked at 70 years in men. Medial joint and patellofemoral joint osteoarthritis were significantly associated with disability (46 v $17 \%$ in controls and $64 v 25 \%$ in controls respectively) but higher HAQ scores were more common in subjects with patellofemoral joint osteoarthritis. Patellofemoral joint osteoarthritis is common, associated with disability, occurs in the absence of tibiofemoral disease, and can no longer be omitted from future studies of osteoarthritis of the knee joint.
\end{abstract}

(Ann Rheum Dis 1992; 51: 844-849)

Osteoarthritis of the knee joint is a common, disabling, and expensive disease which predominantly affects the elderly. ${ }^{12}$ The knee is a complex tricompartmental synovial joint. Despite this studies of osteoarthritis of the knee joint have tended to focus on the tibiofemoral compartment alone. ${ }^{134}$ The patellofemoral compartment, however, is likely to be important.

As a sesamoid bone within the quadriceps femoris tendon, the patella is an integral component of the knee extension apparatus, acting as a dynamic fulcrum transmitting forces of up to eight times the body weight during everyday activities. ${ }^{5}$ Its dual role as an articulation point and a component of the quadriceps muscle makes it unique and suggests that patellofemoral joint osteoarthritis may be a potent cause of knee pain and disability.

Reviews of this subject indicate that patho- logical abnormalities of patellar cartilage are common and increase with age. ${ }^{6}$ Minor changes are common and their relevance to knee pain remains controversial. ${ }^{7} \mathrm{~A}$ distinct subgroup is recognised, however, with more progressive damage usually occurring on the lateral patellar face. ${ }^{6}$ This occurs in older subjects, is more common in women, ${ }^{8}$ and is morphologically indistinguishable from patellofemoral osteoarthritis. It has been observed pathologically ${ }^{6}$ and in radiographic series ${ }^{9}$ that patellofemoral osteoarthritis can occur in the absence of overt tibiofemoral disease. Despite this the patellofemoral joint has received less attention than the tibiofemoral compartment. Its prevalence in the community is not known and the failure to include lateral radiographs in any of the large surveys of knee joint osteoarthritis, ${ }^{134}$ may have contributed to the low correlations observed between symptoms, clinical findings, and radiographic changes. ${ }^{10}$

This study investigated the prevalence of various patterns of radiographic osteoarthritis in subjects living in the community who were asymptomatic and those reporting knee pain.

\section{Subjects and methods}

SUBJECTS

The study group has been described previously. ${ }^{11}$ In brief, a postal questionnaire asking about knee pain and disability was sent to 2101 men and women aged over 55 years registered at a general practice in Bristol. The question about knee pain was taken verbatim from the Health and Nutrition Examination Survey ${ }^{12}:(a)$ have you ever had pain in or around a knee on most days for at least a month? $(b)$ if so, have you had any knee pain during the last year?

A positive response to the two parts of the question was required for a subject to be designated 'knee pain positive'. The response rate to the questionnaire was $81 \%$. Two subgroups of this population were then invited to attend the Bristol Royal Infirmary for clinical assessment of their knee joints and for radiography. These two groups consisted of $(a)$ all knee pain positive respondents and $(b)$ a selection of asymptomatic control subjects.

\section{RADIOGRAPHY}

Weight bearing anteroposterior knee radiographs and recumbent lateral knee radiographs were obtained for all subjects.

The radiographs were scored blindly by the 
same observer (TEM) for the presence of osteoarthritis using the principles of Kellgren and Lawrence, ${ }^{13}$ with joint space narrowing considered mandatory for the designation of definite osteoarthritis. Each received an 'on-off' score with respect to the presence of medial, lateral, and patellofemoral compartment disease as suggested by Ahlback. ${ }^{9}$

\section{Reproducibility}

Fifty sets of knee radiographs were chosen to represent the spectrum of radiographic changes from normal to severe. These were rescored blindly by the original observer. Their relative reproducibility was assessed using the $x$ statistic.

\section{ANALYSIS \\ Compartmental predilection of knee joint osteoarthritis}

The first aim of this study was to determine whether or not osteoarthritis has stereotypic patterns of expression at the knee. It is an analysis which is complicated, however, by the fact that each knee has three compartments and each subject has two knees, generating 49 different patterns of expression of knee joint osteoarthritis per subject. To simplify the process we reduced this part of the analysis to the level of knees rather than subjects. It is therefore important when interpreting the data presented in this paper to be clear about whether the proportions refer to knees or to subjects.

The two subgroups were investigated separately. As the symptomatic group contained a proportion of subjects with only unilateral knee pain the number of knee pain positive knees was not double the number of knee pain positive subjects. These contralateral asymptomatic knees were not included in the analysis of knee pain negative knees because it was felt that they were likely to be unrepresentative of this group.

The proportions of knees with each of the seven potential patterns of osteoarthritis were then calculated separately in the knee pain positive and knee pain negative groups subdivided by age and sex.

Proportion of subjects with knee joint osteoarthritis Subjects were designated as having knee joint osteoarthritis on the basis of radiographic changes in one or two knees. Assigning a compartmental pattern to a subject, however, can be difficult as it is theoretically possible for different patterns to coexist in each knee. Subjects assigned to a category were therefore required to have either unilateral or symmetrical disease, and subjects with mixed patterns were not included.

To assess the extent to which knee osteoarthritis is symmetrical the percentage with bilateral disease from all those with painful knee osteoarthritis was calculated, and the occurrence of radiographic changes in the contralateral asymptomatic knees from knee pain positive subjects was also investigated.

\section{Occurrence of symptomatic patellofemoral joint osteoarthritis in the study group}

This was estimated using the proportions of knee pain positive subjects found to have osteoarthritis together with the data on the occurrence of knee pain derived from the postal questionnaire survey. It provides information about symptomatic disease only.

\section{Disability}

Disability was assessed using the lower limb components of the health assessment questionnaire (HAQ). ${ }^{14}$ The prevalence of disability in subjects with medial or patellofemoral compartment knee osteoarthritis was investigated in a case control manner. Controls were required to be asymptomatic and have radiographically normal knees.

\section{Statistics}

The age and sex distribution of the study sample and knee pain positive subgroup was investigated using the $\chi^{2}$ test. The proportion of subjects with each possible pattern of compartmental disease was determined in the knee pain negative and positive groups stratified by age and gender. Confidence intervals around these proportions and around other proportions reported in this study were derived using the methodology of Gardner and Altman. ${ }^{15}$

\section{Results}

SUBJECTS

A total of 273 knee pain positive subjects and 240 controls attended for radiography. Table 1 presents the detailed breakdown. Compared with the study group minor differences were apparent in the subgroups of men due to over representation of knee pain positive men in the 65-74 year old age group and controls in the 75-84 year old group but this did not reach statistical significance $\left(\chi^{2} 7 \cdot 031\right.$, degrees of

Table 1 Number (percentage) of men and women in the study groups by age

\begin{tabular}{|c|c|c|c|c|c|c|}
\hline \multirow[t]{2}{*}{ Age (vears) } & \multicolumn{2}{|c|}{ Studv group } & \multicolumn{2}{|c|}{ Knec pain positia' group } & \multicolumn{2}{|c|}{ Kne' pain negatiz' group } \\
\hline & Men & Women & $M \bullet n$ & II"ome'n & $M e n$ & I'omen \\
\hline $\begin{array}{l}55-64 \\
65-74 \\
75-84 \\
85+\end{array}$ & $\begin{aligned} 319 & (38) \\
276 & (32) \\
211 & (25) \\
48 & (6)\end{aligned}$ & $\begin{array}{l}289(28) \\
302(30) \\
319(31) \\
107(11)\end{array}$ & $\begin{aligned} 21 & (24) \\
43 & (50) \\
18 & (21) \\
4 & (5)\end{aligned}$ & $\begin{array}{l}54(29) \\
63(34) \\
51 \\
19 \\
19(10)\end{array}$ & $\begin{aligned} 22 & (28) \\
25 & (32) \\
29 & (37) \\
2 & (3)\end{aligned}$ & $\begin{array}{ll}51 & (32) \\
54 & (33) \\
+5 & (28) \\
12 & (7)\end{array}$ \\
\hline Total & 854 & 1017 & 86 & 187 & 78 & 162 \\
\hline
\end{tabular}


freedom $=3, \mathrm{p}<0.1 ; \chi^{2} 4.965, \mathrm{p}<0.1$ respectively). No differences were found in the age or sex distributions of the subgroups of women compared with the study group $\left(\chi^{2} 1 \cdot 112\right.$; 0.547 respectively, degrees of freedom $=3$ ).

One hundred and twenty eight subjects from the knee pain positive group had unilateral symptoms. As the asymptomatic knees were excluded from the analysis of knee joint osteoarthritis patterns this generated 418 knees in the knee pain positive and 480 in the knee pain negative sample.

\section{REPRODUCIBILITY OF RADIOGRAPHS}

Table 2 presents the $x$ values for the designation of compartmental osteoarthritis.

\section{PATTERNS OF KNEE JOINT OSTEOARTHRITIS}

Figure 1 shows the frequency of compartmental patterns of knee joint osteoarthritis in the symptomatic men, symptomatic women, and asymptomatic subgroups respectively. The analysis of knees in these samples showed that from seven potential patterns only three are common: lone medial compartment; lone patellofemoral; and medial/patellofemoral overlap. These are consistent between the samples and between genders. The results of this analysis are summarised in fig 1 .

Any differences which were apparent occurred in the knee pain positive group. Among men with symptomatic knees lone medial compartment disease was the most common pattern, significantly exceeding patellofemoral osteoarthritis overall $(21 v 11 \%, \mathrm{p}<0.05)$. Its apparent frequency increased with age and significantly exceeded the frequency of medial compartment osteoarthritis found in women over the age of $65(\mathrm{p}<0.05)$.

In women with symptomatic knees the converse applied with lone patellofemoral osteoarthritis being more common (24 $v$ 12\% overall; $p<0.05$ ) and tending to increase in frequency with age. Lone medial compartment disease was present in $12 \%$ of knee joints and was not influenced by age.

The third most common pattern, medial/ patellofemoral disease, had similar occurrence in men and women $(7 v 6 \%, \mathrm{NS})$ which tended to increase with age though this did not reach statistical significance.

Other patterns of knee joint osteoarthritis occurred relatively rarely and did not differ between the groups of men and women.

The same patterns were observed in the asymptomatic knee joints but at much lower frequencies. The occurrence of medial and patellofemoral patterns tended to increase with age $(\mathrm{p}<0.05)$ and medial compartment osteoarthritis was approximately twice as common as

Table 2 Reproducibility of radiographic features

\begin{tabular}{ll}
\hline Radiographic feature & $\times$ Statistic \\
\hline Medial compartment osteoarthritis & $1 \cdot 00$ \\
Lateral compartment osteoarthritis & 0.69 \\
Patellofemoral osteoarthritis & 0.61 \\
\hline
\end{tabular}

patellofemoral osteoarthritis. These results did not differ between the subgroups of men and women, which have therefore been presented as a single group in fig 1 .

\section{KNEE JOINT OSTEOARTHRITIS IN THE STUDY} GROUPS

One hundred and forty six knee pain positive subjects (54\%) and 40 asymptomatic subjects (17\%) had evidence of knee joint osteoarthritis. The sensitivity and specificity of knee pain for
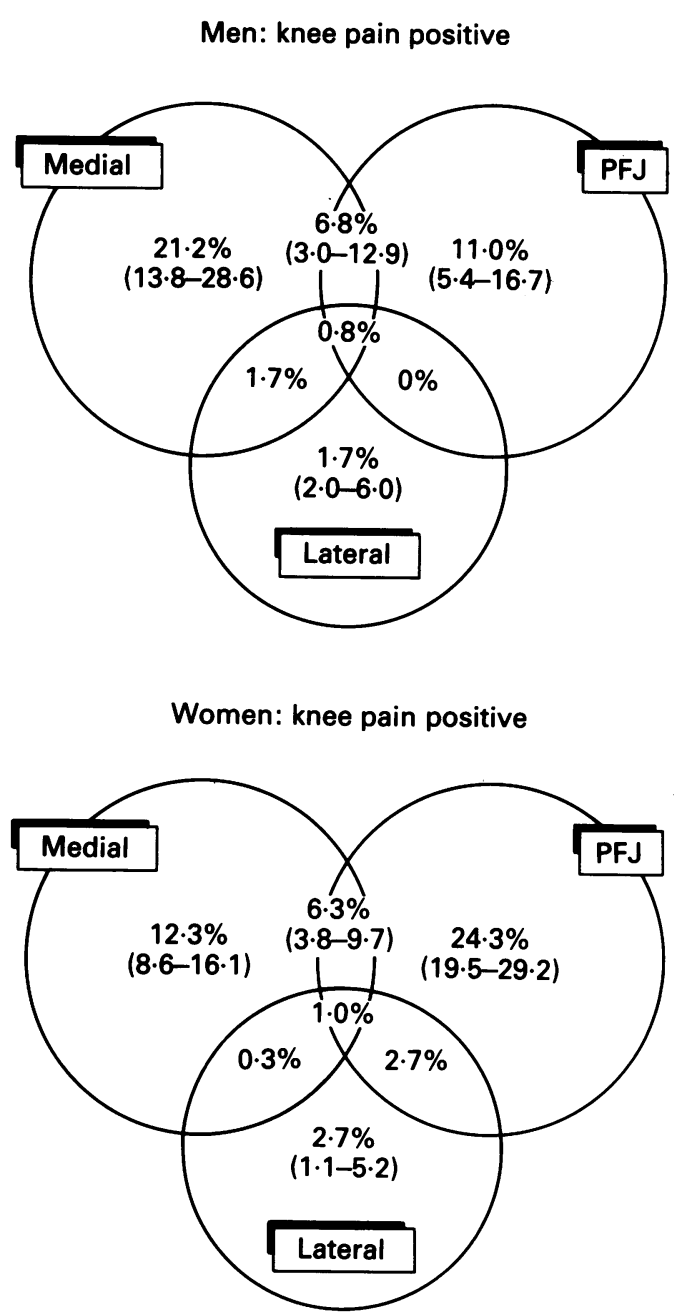

Men and women: knee pain negative

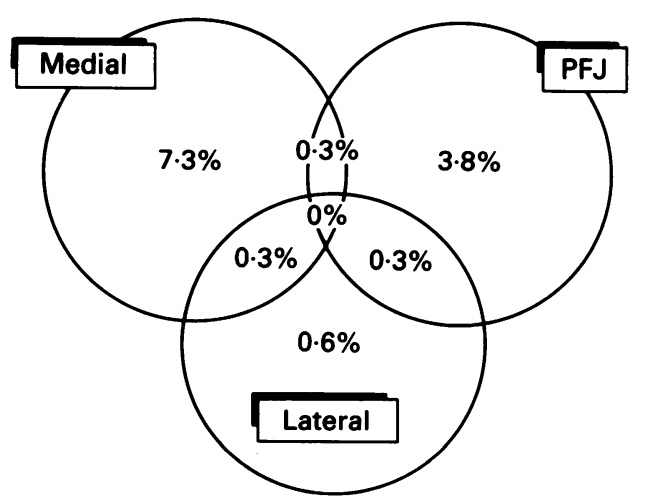

Figure 1 Frequency of compartmental patterns of knee joint osteoarthritis in the symptomatic men and women and the asymptomatic subgroups. $P F F=$ patellofemoral joint . 
radiographic knee joint osteoarthritis were 67 and $70 \%$ respectively.

The proportions of subjects with radiographically detected knee osteoarthritis increased with age in all subgroups; the occurrence of all patterns and of lone patellofemoral osteoarthritis was significantly greater in the knee pain positive group $(p<0.05)$ (tables 3 and 4$)$.

In knee pain positive subjects with knee joint osteoarthritis approximately half of the subgroup of men and three quarters of the subgroup of women had disease in both knees (see table 5).

Table 3 Percentages of subjects with knee joint osteoarthritis (all patterns), lone patellofemoral osteoarthritis, and lone medial compartment knee osteoarthritis among the study samples. Men and women in the knee pain negative sample have been amalgamated due to small numbers. $95 \%$ confidence intervals are given in parentheses

\begin{tabular}{llllll}
\hline $\begin{array}{l}\text { Patterns of knee } \\
\text { joint osteoarthritis }\end{array}$ & \multicolumn{2}{l}{ Knee pain positive } & \multicolumn{2}{l}{ Knee pain negative } \\
\cline { 2 - 3 } \cline { 5 - 6 } & Men & Women & Men & Women \\
\hline All & $48(38$ to 59$)$ & $56(48$ to 63$)$ & & $15(8$ to 25$)$ & $17(11$ to 23$)$ \\
Patellofemoral & $12(5$ to 20$)$ & $26(20$ to 33$)$ & $5(2$ to 8$)$ & $5(2$ to 8$)$ \\
Medial & $23(15$ to 34$)$ & $14(9$ to 20$)$ & $9(6$ to 11$)$ & $9(6$ to 11$)$ \\
\hline
\end{tabular}

Table 4 Proportions of subjects with knee joint osteoarthritis, lone medial, and lone patellofemoral patterns, in the knee pain positive subset according to age and sex. $95 \%$ confidence intervals are given in parentheses

\begin{tabular}{|c|c|c|c|}
\hline Age (vears) & All patterns & Medial & Patellofemoral \\
\hline $\begin{array}{c}\text { Men } \\
55-64 \\
65-74 \\
75+\end{array}$ & $\begin{array}{l}24(8 \cdot 2 \text { to } 47 \cdot 2) \\
53(37 \cdot 7 \text { to } 88 \cdot 8) \\
64(40 \cdot 7 \text { to } 82 \cdot 8)\end{array}$ & $\begin{array}{l}10(1 \cdot 7 \text { to } 27 \cdot 1) \\
28(17 \cdot 0 \text { to } 41 \cdot 3) \\
27(12 \cdot 6 \text { to } 33 \cdot 6)\end{array}$ & $\begin{array}{r}14(4.0 \text { to } 32.9) \\
12(5.0 \text { to } 22.9) \\
9(1.6 \text { to } 25.9)\end{array}$ \\
\hline $\begin{array}{c}\text { Women } \\
55-64 \\
65-74 \\
75-84 \\
85+\end{array}$ & $\begin{array}{l}39(25 \cdot 9 \text { to } 53 \cdot 1) \\
59(45 \cdot 6 \text { to } 71 \cdot 0) \\
59(44 \cdot 2 \text { to } 72 \cdot 4) \\
84(60 \cdot 4 \text { to } 96 \cdot 6)\end{array}$ & $\begin{array}{l}19(10.4 \text { to } 29 \cdot 4) \\
14(7.7 \text { to } 23 \cdot 6) \\
10(3.9 \text { to } 19.5) \\
16(4.4 \text { to } 35 \cdot 9)\end{array}$ & $\begin{array}{l}17(9 \cdot 0 \text { to } 27 \cdot 3) \\
30(20 \cdot 7 \text { to } 41 \cdot 0) \\
31(20 \cdot 8 \text { to } 43 \cdot 7) \\
26(11 \cdot 0 \text { to } 47 \cdot 6)\end{array}$ \\
\hline
\end{tabular}

Table 5 Proportions of subjects with bilateral disease from the subsample with symptomatic knee joint osteoarthritis. 95\% confidence intervals are given in parentheses

\begin{tabular}{lll}
\hline Age (years) & Men & Women \\
\hline $55-64$ & $20(0 \cdot 5$ to $71 \cdot 6)$ & $76(52 \cdot 8$ to $91 \cdot 8)$ \\
$65-74$ & $52(30 \cdot 6$ to $73 \cdot 2)$ & $70(53.0$ to 84.1$)$ \\
$75+$ & $64(35.1$ to $87 \cdot 2)$ & $74(58.9$ to 85.7$)$ \\
\hline
\end{tabular}
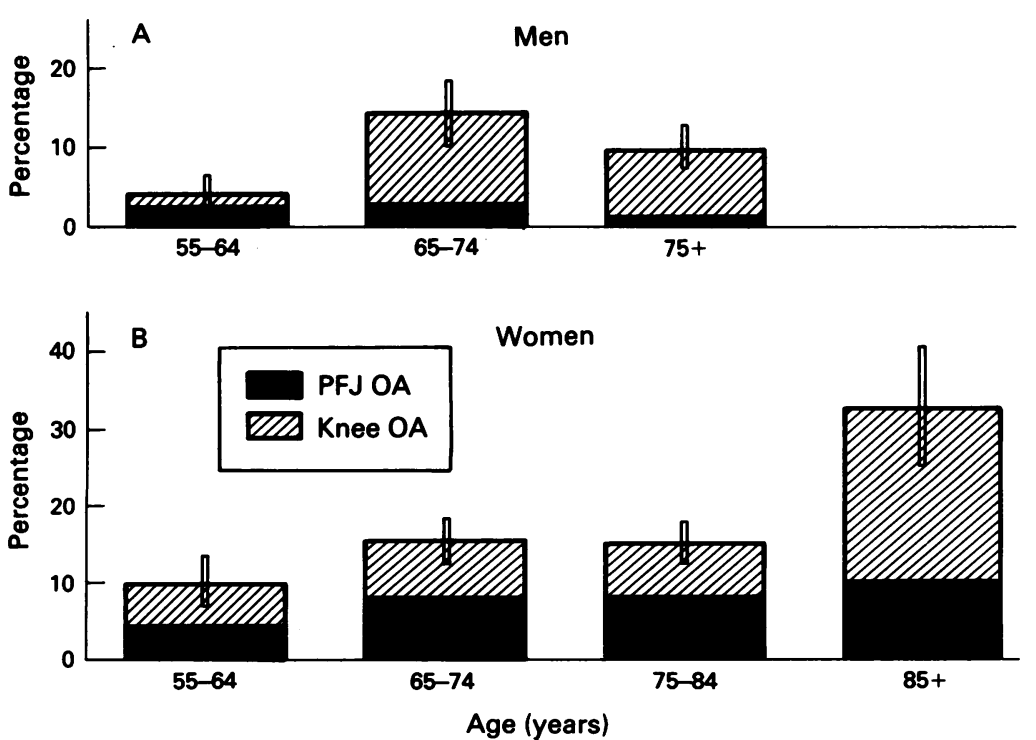

Figure 2 Prevalence of knee joint osteourthritis in the studv group. $P F Y=$ patellofemoral joint.
Table 6 Proportions of subjects with medial and patellofemoral osteoarthritis (OA) reporting disability compared with controls. 'Any disability' is equivalent to a Health Assessment Questionnaire score $>0$, 'severe' disability $\geqslant 2.95 \%$ confidence intervals are given in parentheses

\begin{tabular}{|c|c|c|}
\hline Subject group & $\begin{array}{l}\text { Percentage with } \\
\text { any disability }\end{array}$ & $\begin{array}{l}\text { Percentage with } \\
\text { severe disability }\end{array}$ \\
\hline $\begin{array}{l}\text { Medial compartment OA } \\
\text { Controls } \\
\text { Patients } \\
\text { Ratio }\end{array}$ & $\begin{array}{c}18(8 \cdot 2 \text { to } 31 \cdot 8) \\
46(33 \cdot 4 \text { to } 59 \cdot 1) \\
2 \cdot 6\end{array}$ & $\begin{array}{c}5(1.0 \text { to } 13 \cdot 3) \\
13(5.6 \text { to } 32.5) \\
2.6\end{array}$ \\
\hline $\begin{array}{l}\text { Patellofemoral OA } \\
\text { Controls } \\
\text { Patients } \\
\text { Ratio }\end{array}$ & $\begin{array}{c}25(14 \cdot 4 \text { to } 38 \cdot 4) \\
64(50 \cdot 4 \text { to } 76 \cdot 6) \\
2 \cdot 6\end{array}$ & $\begin{array}{c}4(0 \cdot 4 \text { to } 12 \cdot 3) \\
16(7 \cdot 6 \text { to } 28 \cdot 3) \\
4 \cdot 5\end{array}$ \\
\hline
\end{tabular}

Further evidence of the bilateral nature of this disease was provided by the contralateral knee joints from 128 subjects with unilateral knee pain. Over $50 \%$ of these had radiographically detected osteoarthritis, significantly more $(p<0.05)$ than was found in the asymptomatic group. Furthermore all but $12 \%$ of subjects with knee pain osteoarthritis could be assigned to a compartmental pattern by the presence of either unilateral or symmetrical disease.

PREVALENCE OF KNEE JOINT OSTEOARTHRITIS IN THE COMMUNITY

The estimated prevalences of symptomatic medial, patellofemoral, and medial/patellofemoral knee joint osteoarthritis, extrapolated from the knee pain positive study sample and from knowledge of the occurrence of knee pain in various age ranges in the study population, are presented in fig $2 \mathrm{~A}$ and $\mathrm{B}$.

DISABILITY RATES AND RADIOGRAPHIC PATTERNS Age and sex matched controls were found for 63 subjects with medial and 56 with patellofemoral compartment knee joint osteoarthritis. The groups were mutually exclusive and the numbers of subjects with other patterns were insufficient for analysis. Subjects with either medial or patellofemoral compartment knee osteoarthritis had significantly $(\mathrm{p}<0.01)$ higher occurrences of reported disability (table 6 ). The prevalence of disability in subjects with medial compartment disease was 2.6 times that of their control subjects. This ratio remained constant when higher levels of disability (HAQ score greater than one or two) were considered. For patellofemoral compartment osteoarthritis the ratio was $3 \cdot 1$ overall and increased for these higher levels of disability.

\section{Discussion}

We believe that this is the first study to show the prevalence of patellofemoral osteoarthritis in the community. The condition is common and associated with disability. As a cause of severe disability it may be even more important than medial compartment knee osteoarthritis. The most important conclusion of this paper is that epidemiological and clinical studies of knee osteoarthritis can no longer be performed 
without consideration of the patellofemoral compartment.

One of the main difficulties encountered in this study related to the development of a radiographic scoring system. Previous work in this field ${ }^{134}{ }^{10}$ has relied on a system adapted from the original standard knee radiographs of Kellgren and Lawrence. ${ }^{13}$ These, however, do not include the patellofemoral compartment and investigations of the relative reproducibility of the radiographic features of osteoarthritis in different compartments of the knee joint suggest increased variability in scoring patellofemoral osteoarthritis. ${ }^{16}$

The radiographs in this study were therefore scored by the same observer using an 'on-off' measure as opposed to a severity scale. The reproducibility of this score in each of the three compartments was re-evaluated using the $x$ statistic. The results were favourable using this method, although the $x$ statistic for patellofemoral osteoarthritis compartment was lower than that for medial or lateral compartment osteoarthritis. In contrast with the tibiofemoral joint little attention has been paid to the development of a scoring system for patellofemoral osteoarthritis, and this therefore is at an earlier stage of evolution. As no better system currently exists, our 'on-off' score was considered adequate for use in this study. For future research further work is needed to optimise a system with which to grade the severity of patellofemoral osteoarthritis.

The inclusion of patellofemoral imaging in this survey appears to improve concordance between knee pain and radiographic changes. Claessens et al showed a sensitivity, specificity, and predictive value of knee pain against radiographic osteoarthritis of 24,90 , and $36 \%$ respectively ${ }^{10}$ (see table 7 ). Our results show improvements in the sensitivity and predictive value, probably as a result of detecting subjects with predominant patellofemoral osteoarthritis which would have been missed using anteroposterior radiographs alone.

Specificity is decreased, however, which suggests that other differences are operative between the scoring systems. For example, it is likely that the threshold for the designation of tibiofemoral osteoarthritis was higher in this study than in the work of Claessens et al ${ }^{10}$ and this could account for a reduction in specificity by reducing the proportion of true negative cases.

The next problem related to the large number of potential patterns of knee joint osteoarthritis per subject. To simplify the process of pattern analysis within each knee joint, this part of the study was conducted at the levels of knees rather than subjects. This manoeuvre was helpful in indicating the most common compartmental predilection of osteoarthritis within the knee joint, but as the disease cannot automatically be

Table 7 Knee pain as a predictor of knee osteoarthritis

\begin{tabular}{lll}
\hline Parameter & McAlindon et al " Claessens et al ${ }^{\prime \prime}$ \\
\hline Sensitivity (\%) & $67 \cdot 3$ & 24 \\
Specificity (\%) & $70 \cdot 1$ & 90 \\
Predictive value (\%) & $47 \cdot 8$ & 36 \\
\hline
\end{tabular}

assumed to be symmetrical it is important to be clear whether the data relate to proportions of knees or proportions of subjects. It showed that, despite a large number of potential compartmental patterns, medial and patellofemoral patterns predominate. They appear to be relatively exclusive but with a small potential for overlap. Other patterns seem rare.

Within the pooled subgroup of painful knees the occurrence of medial compartment knee osteoarthritis appears to increase with age in men whereas patellofemoral disease increases in women. As these figures relate to percentages of knees it cannot necessarily be inferred that the percentages of subjects with these patterns also increase. For example, it is possible that the increase in medial compartment disease in men results from increased bilaterality with increasing age rather than an increase in the number of subjects with the disease. The rates of bilateral disease seen in knee pain positive men supports this (table 4).

A broader picture of the nature of knee joint osteoarthritis can be achieved by looking at the knee pattern data together with the information about bilaterality and the proportions of subjects found to have osteoarthritis. In men these suggest a trend of increasing bilaterality with age in what is predominantly medial compartment disease. The increase in occurrence of overall knee osteoarthritis appears to be accountable to mixed patterns of expression as the rates of medial, medial/patellofemoral, and patellofemoral patterns do not alter in men after the age of 65 . In women neither the bilaterality rates nor the occurrence of medial or patellofemoral disease increases after 65 years. This suggests that the increase in overall knee joint osteoarthritis is attributable to a genuine increase in the numbers of subjects with medial/ patellofemoral or mixed patterns of expression.

These patterns may either reflect different disease processes or a similar predisposition with various risks or environmental factors resulting in different patterns of expression. It is tempting to speculate that the increase in the medial/patellofemoral pattern in women is due to patellar problems developing in subjects with established medial compartment disease as a result of tracking abnormalities. If so, this could contribute to the apparent plateau in the occurrence of lone medial disease in women. It is, however, difficult to address cause and effect relations in a cross sectional survey such as this.

An attempt to estimate the prevalence of symptomatic knee joint osteoarthritis in the study group was made based on the occurrence found in the knee pain positive subgroup and the known knee pain rates derived from the postal questionnaire survey. There are a number of problems with this. Firstly, the figures refer to symptomatic disease only, and as such are inevitably an underestimate of the overall prevalence. Also it is possible that knee osteoarthritis is associated with comorbidity. The issue of coexistent disease was not addressed in this work, but if present might be a source of bias at the level of response to the postal questionnaire and with respect to attendance for 
assessment. Such bias might in part explain the unusual finding of an apparent decrease in the prevalence of knee joint osteoarthritis after the age of 70 years in men. Alternative explanations are that a cohort effect is operative (though a similar decrease in prevalence might have been expected in women) or the influence of selective mortality for which there is some evidence. ${ }^{17} 18$ These factors, together with the possibility that a higher threshold for the designation of knee joint osteoarthritis was adopted in this study, may account for the lower estimated prevalence found here than in previous surveys. ${ }^{14}$

Finally both medial and patellofemoral osteoarthritis are strongly associated with disability. Higher levels of disability (as judged by HAQ scores of greater or equal to one or two) seem more common in subjects with patellofemoral disease, a plausible result given the close anatomical and functional relation of the patella to the quadriceps apparatus.

In summary, patellofemoral disease is a common manifestation of knee joint osteoarthritis. It is often bilateral, is significantly associated with pain and disability, and can no longer be disregarded in studies of knee joint osteoarthritis.

We thank Dr I Watt, Department of Radiology, Bristol Royal Infirmary for his advice and help with this study, Dr T Paine and partners, Whiteladies Health Centre for their collaboration, and partners, Whiteladies Health Centre for their collaboration, and
finally their patients, without whose willing cooperation this finally their patients, without whose willing cooperation this
work would not have been possible. This project was funded by the Arthritis and Rheumatism Council.
1 Van Saase J L C M, Van Romunde L K J, Cats A, Vandenbroucke J P, Valkenburg $\mathrm{H}$ A. Epidemiology of osteoarthritis: Zoetermeer survey. Ann Rheum Dis 1989; 48: 271-80.

2 Wright V. Osteoarthritis. BMF 1989; 299: 1476-7.

3 Lawrence J S, Bremner J M, Bier F. Osteoarthrosis: prevalence in the population and relationship between prevalence in the population and relationship between
symptoms and x-ray changes. Ann Rheum Dis 1966; 25: symptom.

4 Felson D T, Naimark A, Anderson J, Kazis L, et al. The prevalence of knee osteoarthritis in the elderly. Arthritis Rheum 1987; 30: 914-8.

5 Hungerford D S, Barry M. Biomechanics of the patellofemoral ioint. Clin Orthop Rel Res 1979; 144: 9-15.

6 Meachim G. Cartilage lesions on the patella. In: Pickett J C, Radin E L, eds. Chondromalacia of the patella. Baltimore: Williams \& Wilkins, 1983.

7 Cascells W. Chondromalacia patellae and its relationship to anterior femoral pain. In: Pickett J C, Radin E L, eds. Chondromalacia of the patella Baltimore: Williams \& Wilkins, 1983 .

8 Meachim G, Pedley R B. Implications of a sex difference in osteoarthrosis. Ann Rheum Dis 1980; 39: 199.

9 Ahlbach S. Osteoarthritis of the knee: a radiographic investigation. Acta Radiologica Suppl 1968; 277: 1-61.

10 Claessens A A M C, Schouten J S A G, Van den Ouweland F A, Valkenburg H A. Do clinical findings correlate with osteoarthritis of the knee? Ann Rheum Dis 1990; 49: 771-4.

11 McAlindon T E, Cooper C, Dieppe P. Knee pain and disability in the community. Br $\mathcal{Y}$ Rheumatol 1990; 29 (suppl 2): 87 .

12 National Center for Health Statistics. Basic data on arthritis of the hip, knee and sacro-iliac joints in adults ages 25-74, United States, 1971-1975. Series 11, No 213, 1979.

13 Kellgren J H, Lawrence J S. Radiological assessment of osteoarthrosis. Ann Rheum Dis 1957; 16: 494-501.

14 Fries J F, Spitz P, Young D Y. The dimensions of health outcomes: the Health Assessment Questionnaire, disability outcomes: the Health Assessment Questionnaire,

15 Gardner M J, Altman D G, eds. Calculating confidence intervals for proportions and their differences. Statistics with confidence. London: BMJ, 1989: 28-33.

16 Cooper C, Cushnaghan J, Kirwan J R, et al. Radiographic assessment of the knee joint in osteoarthritis. Ann Rheum Dis. 1992; 51: 80-2.

17 Bergstrom G, Bjelle A, Sundh V, Svanborg A. Joint disorders at ages 70,75 and 79 years - a cross sectional comparison. at ages 7 , Reumatol 1986; 25: 333-41.

18 Hochberg M C, Lawrence R C, Everet D F, CornoniHuntley J. Epidemiologic associations of pain in osteoarthritis of the knee. Semin Arthritis Rheum 1989; 18: (supp
and 2): $4-9$. 